\title{
Correction: Glaucoma in large-scale population-based epidemiology: a questionnaire-based proxy
}

\author{
Anna Neustaeter $(\mathbb{D} \cdot$ Jelle Vehof $\cdot$ Harold Snieder $(\mathbb{D} \cdot$ Nomdo M. Jansonius
}

Published online: 30 March 2021

(c) The Author(s), under exclusive licence to The Royal College of Ophthalmologists 2021

\section{Correction to: Eye}

https://doi.org/10.1038/s41433-020-0882-4

The original version of this article unfortunately contained a mistake. The Acknowledgements section was incorrect. The corrected Acknowledgements section is given below. The original article has been corrected.
Acknowledgements This project has received funding from the European Union's Horizon 2020 research and innovation programme under the Marie Skłodowska-Curie grant agreement EGRET No 661883. The funding organisation had no role in the design, conduct, analysis, or publication of this research. The Lifelines Biobank initiative has been made possible by subsidy from the Dutch Ministry of Health, Welfare and Sport, the Dutch Ministry of Economic Affairs, the University Medical Center Groningen (UMCG the Netherlands), University of Groningen and the Northern Provinces of the Netherlands. 